\title{
Coincidence of juvenile idiopathic arthritis and type 1 diabetes: a case-based review
}

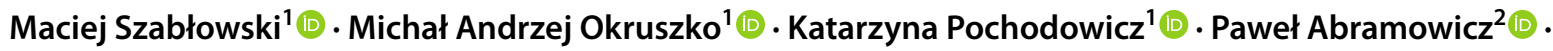 \\ Jerzy Konstantynowicz ${ }^{2}\left(\right.$ Artur Bossowski $^{1}$ (1) B Barbara Głowińska-Olszewska ${ }^{1}(\mathbb{C}$
}

Received: 25 October 2021 / Accepted: 28 December 2021 / Published online: 8 January 2022

(c) The Author(s) 2022

\begin{abstract}
The study was aimed to review a rare coexistence of type 1 diabetes (T1D) and juvenile idiopathic arthritis (JIA) regarding different clinical approaches to the management and treatment options. Medical complications of the two autoimmune disorders in children and adolescents have been evaluated, particularly in those treated with glucocorticosteroids (GCS) and insulin. A review of the literature regarding reports on concomitant T1D and JIA was conducted using resources available in Medline, Google Scholar, and Web of Science databases, with a specific focus on the combination of T1D and JIA in a pediatric population. The review was extended by our analysis of two patients treated in a single center for this comorbidity. Eligible reports of four cases were found, and including our two original records, a total of six pediatric patients (5 females) were analyzed, of which three had also other autoimmune diseases (thyroiditis, coeliac disease, autoimmune hepatitis), whereas four had been treated with a long-term GCS, and two were receiving biological therapy (etanercept or adalimumab). Only one of them had good metabolic control of diabetes. Diabetes in childhood may coexist with other autoimmune diseases, including rheumatologic conditions. Hyperglycemia can worsen JIA therapy by induction and maintaining inflammation. Using modern diabetes technologies (like personal insulin pumps and continuous glucose monitoring) helps to minimize the deteriorating effect of JIA exacerbations and the rheumatoid treatment on metabolic control of diabetes.
\end{abstract}

Keywords Autoimmune diseases $\cdot$ Children $\cdot$ Comorbidity $\cdot$ Diabetes mellitus type $1 \cdot$ Juvenile arthritis

$\begin{array}{ll}\text { Abbreviations } \\ \text { T1D } & \text { Type 1 diabetes mellitus } \\ \text { JIA } & \text { Juvenile idiopathic arthritis } \\ \text { GCS } & \text { Glucocorticosteroids } \\ \text { DMARD } & \begin{array}{l}\text { Disease-modifying antirheumatic drug } \\ \text { ISPAD }\end{array} \\ \begin{array}{l}\text { International Society for Pediatric and Ado- } \\ \text { lescent Diabetes }\end{array} \\ \text { ILAR } & \begin{array}{l}\text { International League of Associations for } \\ \text { Rheumatology }\end{array} \\ \text { CSII } & \begin{array}{l}\text { Continuous subcutaneous insulin infusion } \\ \text { CGM }\end{array} \\ \text { Continuous glucose monitoring system } \\ \text { PIP } & \text { Proximal interphalangeal joint }\end{array}$

Barbara Głowińska-Olszewska

barbara.glowinska-olszewska@umb.edu.pl

1 Department of Pediatrics, Endocrinology, Diabetology With Cardiology Division, Medical University of Białystok, Białystok, Poland

2 Department of Pediatrics, Rheumatology, Immunology and Metabolic Bone Diseases, Medical University of Białystok, Białystok, Poland

$\begin{array}{ll}\text { DIP } & \text { Distal interphalangeal joint } \\ \text { MCP } & \text { Metacarpophalangeal joint } \\ \text { ESR } & \text { Erythrocyte sedimentation rate } \\ \text { JADAS } & \text { Juvenile Arthritis Disease Activity Score } \\ \text { RF } & \text { Rheumatoid factor } \\ \text { ACPA } & \text { Anti-citrullinated peptide antibody } \\ \text { TNF-alpha } & \text { Tumor necrosis factor-alpha } \\ \text { FGM } & \text { Flash glucose monitoring system } \\ \text { HLA } & \text { Human leukocyte antigen }\end{array}$

\section{Introduction}

Diabetes is regarded as a global health problem in the twenty-first century. The incidence of type 1 diabetes (T1D) has increased by $3.4 \%$ during recent decades, and the continuing rise is predicted, which confers a severe public health burden worldwide [1-4]. Some commonly observed comorbidities of T1D, like Hashimoto thyroiditis and coeliac disease, are well-known, and proper screening programs at the diagnosis of diabetes are established. Nevertheless, other 
conditions like juvenile idiopathic arthritis (JIA) and Addison's disease remain less explored, so clinicians involved in diabetes care might also expect some revisited algorithms or screening tools concerning the comorbidity.

A cluster of multiple diseases in the same person implicates several clinical, social, and therapeutic issues, often leading to complications and challenging treatment decisions. The problem may emerge because of different symptoms, of which co-occurrence obscures the clinical picture of individual disease entities, making it difficult to diagnose due to polypharmacy. This manifests itself concerning autoimmune diseases in the treatment of which pleiotropic and multitargeted drugs are used. An example of such a disease is JIA. The literature shows that patients incurring this disease are at higher risk of T1D. Some research suggests that it is associated with the mutation in the PTPN22 gene encoding an enzyme inhibiting the T-cell activation pathway $[5,6]$. Both the rheumatologic condition per se and a long-lasting treatment burden may significantly negatively impact T1D. Inflammation as a core element of JIA and glucocorticosteroids (GCS) use exacerbate glycemia fluctuations making it challenging to determine the insulin doses and keep them constant. A cumulative effect of those factors disturbs the diabetes metabolic control [7].

JIA and T1D are also significant public health issues in pediatric populations worldwide; two chronic diseases with the early onset in childhood, resulting in short-term and late, life-long consequences and complications, with a powerful impact on health status in adulthood. Therefore, we intended to review the contemporary therapeutic approach to these diseases and point out specific difficulties in the course of treatment, based on two cases of patients with comorbid JIA and T1D from our center, extended by the analysis of another case series reported in commonly used databases.

\section{Search strategy}

The literature search in the electronic databases: Medline (via PubMed), Web of Science, and Google Scholar was conducted in March 2021. We used the entry keywords "Arthritis, Juvenile" AND "Diabetes Mellitus, Type 1" using MeSH thesaurus on PubMed. When searching Google Scholar and Web of Science databases, we were using "Juvenile idiopathic arthritis" AND "diabetes mellitus type 1" AND "case study" OR "case report" terms. We included the studies published from 2010 until the day of the search, published in English language and concerning pediatric patients diagnosed with both the T1D (diagnosis based on International Society for Pediatric and Adolescent Diabetes (ISPAD) [8]) and JIA (meeting the International League of Associations for Rheumatology, ILAR) classification criteria [9]). We reviewed the abstracts of the relevant studies and subsequently retrieved the appropriate articles. We also scrutinized the reference lists of the included studies to identify additional references. Four case studies that met the adopted criteria were found [10-13]. Furthermore, we reported the history of two children treated in an academic tertiary care hospital; medical records, direct examination, and clinical assessment were used. The data and summary of clinical and laboratory results of all six eligible cases are shown in Table 1.

\section{Original case 1}

An 18-month-old girl was admitted to the hospital because of low-grade fever, rhinitis, and cough for 2 days. She was transferred from the infectious diseases department to our endocrine unit because of hyperglycemia. During the week preceding the hospitalization, the girl manifested polyuria, polydipsia, $2 \mathrm{~kg}$ weight loss. On admission, she was lethargic and vomited. Hyperglycemia of $711 \mathrm{mg} / \mathrm{dL}(39.5 \mathrm{mmol} / \mathrm{L})$ and ketoacidosis $(\mathrm{pH}=7.31$, base excess $=-16)$ were reported. Physical examination revealed the features of dehydration and Kussmaul breathing with acetone odor. She was diagnosed with type 1 diabetes. Initial treatment included intravenous insulin and rehydration, resulting in normoglycemia. Subcutaneous insulin therapy and a diabetic diet were implemented from the second day of hospitalization. Because of her young age, the decision was made to start a continuous subcutaneous insulin infusion (CSII) with a personal insulin pump system from the very beginning. Further laboratory investigation proved glycated hemoglobin HbA1c of $10.63 \%$, low serum 25-hydroxyvitamin D concentration. According to the current recommendations, screening for autoimmune thyroid diseases and coeliac disease was carried out with a negative result [14]. After 5 days of insulin treatment, new symptoms appeared: swollen right knee (with limping), and after the next 9 days-swelling of the interphalangeal joints of the left hand. Treatment with an anti-inflammatory drug orally, topically, and intravenous antibiotic therapy (amoxicillin-clavulanate) was started. The musculoskeletal ultrasound of the affected articulations revealed joint effusion and synovial hypertrophy with concomitant tenosynovitis of flexors. Due to the spreading inflammatory process, a small dose of prednisone was used with a good response. Upon specialist advice by the rheumatologist, subsequent laboratory findings showed an increased erythrocyte sedimentation rate, lack of rheumatoid factor (RF), and anticitrullinated protein antibody (ACPA). After her parents were educated in insulin therapy and diabetes management, the patient was discharged home and referred to the rheumatologic follow-up. Two months following the initial hospitalization, an experienced rheumatologist made a definite JIA RF-negative diagnosis, according to ILAR criteria (second revision) [9]. The treatment with a small 


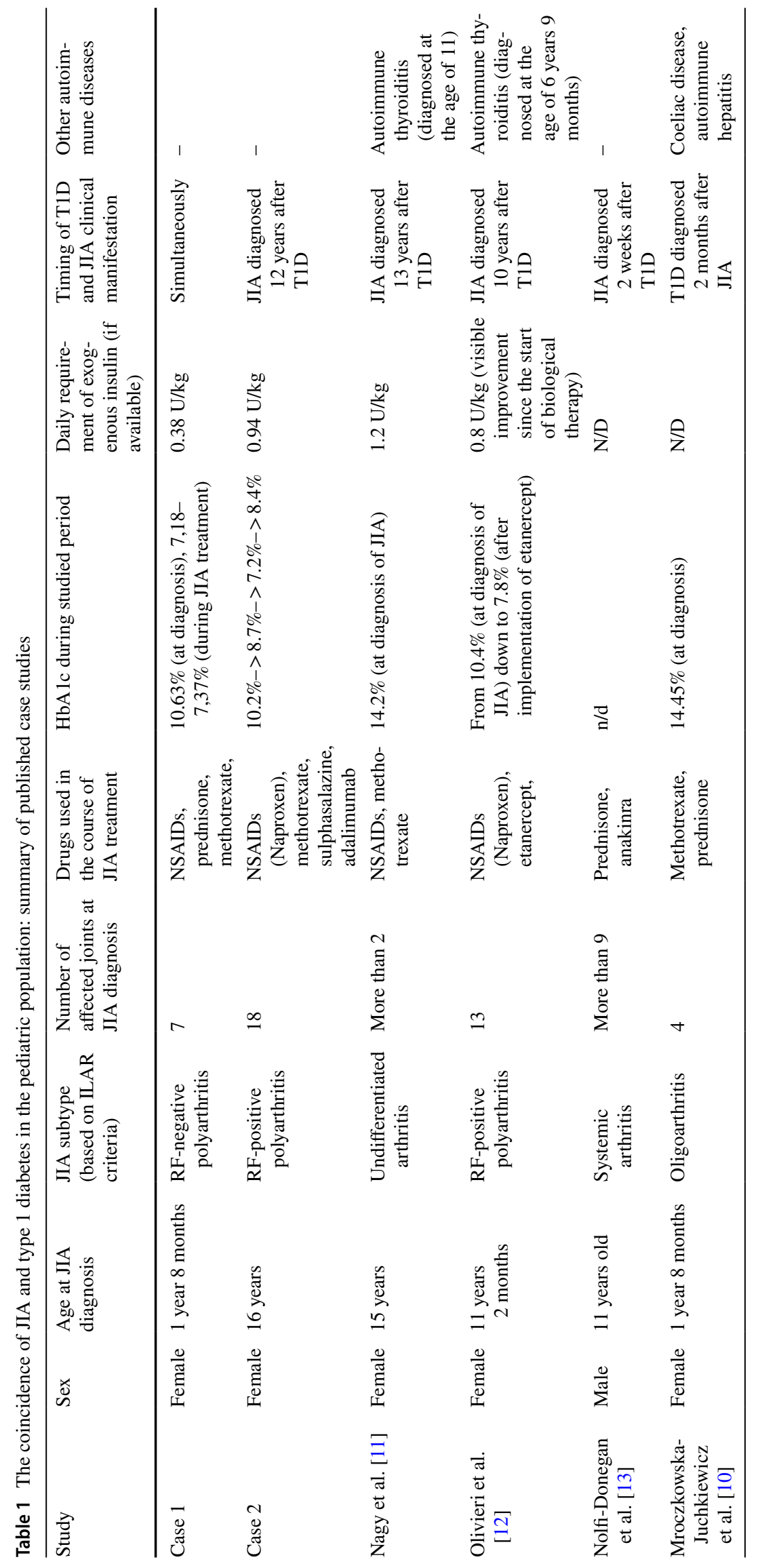


dose of oral GCS was maintained. Improvement was noted during the follow-up visit: she had normal physical activity, but walking difficulty with limited range of movement in the lower extremity persisted. The affected joints (right knee and proximal interphalangeal (PIP), distal interphalangeal (DIP), metacarpophalangeal (MCP) joints of the left II and III fingers) remained slightly swollen; however, the range of motion was full. Due to the number of involved joints, the treatment with intraarticular glucocorticoid injections was not implemented. A small dose of oral GCS $(0.3 \mathrm{mg}$ per $\mathrm{kg}$ of body weight of prednisone) was repeated, and oral methotrexate in the weekly dose of $10 \mathrm{mg} /$ week was initiated, with further tapering and discontinuation of steroids. Disease remission was achieved in accordance with Wallace criteria [15]. Despite a few viral infections and the GCS use, the glycemic values were kept within an acceptable range (HbA1c of 7.1-7.32\%). A continuous glucose monitoring system (CGM) was implemented to improve glycemic control.

The CGM consists of a sensor applied under the skin and measuring glucose level in the interstitial fluid, transmitter, and a display device (e.g., dedicated application on the smartphone) that continuously collects data from the sensor, enabling the patient to know their approximate current glycemia. After advanced educational training, parents were using CGM actively with a noticeable improvement of metabolic control of diabetes.

The above example shows that patients happen to be diagnosed with more than just diabetes from the very beginning, also at a very young age. Insulin therapy of such a child is a significant therapeutic challenge for both the medical team and caregivers and thus, requires close interactive cooperation.

\section{Original case 2}

A 16-year-old girl with an 11-years-lasting history of T1D was admitted to the rheumatology unit because of chronic pain and swelling of multiple joints (knees, ankles, hips, shoulders, temporomandibular joints, of varying degrees of severity) having persisted for the previous two months. She had been treated with NSAIDs with no improvement. Based on the overall clinical picture, ultrasound examination, the results of laboratory findings (increased CRP, ESR), the presence of RF and ACPA, the diagnosis of polyarticular seropositive JIA was established according to ILAR criteria [9]. The treatment with oral methotrexate was started in an increasing weekly dose up to $15 \mathrm{mg} / \mathrm{m}^{2}$ (switched to subcutaneous administration due to poor tolerability of oral treatment). Due to the persistence of high disease activity, expressed as JADAS (Juvenile Arthritis Disease Activity Score) value of 14.8 , sulphasalazine, another disease-modifying antirheumatic drug (DMARD), was added after 3 months [16]. Despite that 3-months-long combined therapy with two conventional DMARDs, response to treatment was insufficiently attained (JADAS 9.7); therefore, subcutaneous tumor necrosis factor-alpha (TNF-alpha) inhibitor (adalimumab) was started. During the initial phase of DMARD treatment, a small dose of oral glucocorticoids $(0.5 \mathrm{mg} / \mathrm{kg}$ of body weight of prednisone) were used as 'bridge' therapy to achieve a quick alleviation while awaiting the full therapeutic effect of DMARDs. Afterward, due to JIA flares, oral GCS were continued temporarily. Her metabolic control of diabetes during the JIA treatment deteriorated much at first, resulting partly from poor compliance. The patient measured blood glucose rarely (3-4 times a day) and did not use the bolus calculator. Her blood glucose reached an average value of over $200 \mathrm{mg} \%$ (with a standard deviation over 100). She was covered by the assistance of a clinical board-certified psychologist (specialized in the management of chronic diseases) on the regular meeting sessions using individual psychotherapy. Psychological sessions were mainly aimed at strengthening acceptance of her chronic conditions, increasing her self-esteem, and motivating her to achieve the therapeutic goals gradually. She also met, on a regular basis, with specialized diabetes educators who taught her to respond appropriately to various scenarios in everyday life, to prevent hypo- and hyperglycemia. Along with intensive diabetes care, systematic reeducation, psychological support, and the use of a flash glucose monitoring system (FGM), stepwise amelioration in $\mathrm{HbA} 1 \mathrm{c}$ from 10.23 to $7.2 \%$ was observed. The insulin requirements of this patient throughout her JIA treatment ranged from 0.92 to $0.94 \mathrm{U} /$ $\mathrm{kg}$. However, further exacerbations of the JIA and temporary use of oral GCS still worsened the diabetes control; the girl presented with $\mathrm{HbA1c}$ over $8 \%$. In this patient, CSII combined with FGM were crucial. The implementation of the FGM system, 1 month after the patient had commenced adalimumab, allowed her to manage better the glycemic variability. Regular FGM use alleviated the complaints and laboratory indicators: the percentage of data collected from the sensor reached $87 \%$, whereas the number of boluses per day increased from 6 to 9 . The girl had to change insulin dosage every 2-3 days because of changing steroid treatment, adjusted to her rheumatologic disease. It remains a challenging situation for her because of persistent joint complaints and a decreased quality of life caused by blood glucose fluctuations. The combined treatment made it possible to achieve partial remission before she was transferred to an adult clinic (JADAS score of 4.5).

To summarize, insulin therapy in the adolescent patient was challenging, considering teenage non-compliance and reluctance to cope with the disease. In addition, the GCS therapy may have considerably constricted effective metabolic control of the patient. Furthermore, a significant clinical issue was an inadequate response to treatment of JIA, 
resulting in numerous modifications, including ultimately the initiation of biologic therapy.

\section{Discussion}

T1D is the most common chronic metabolic disease of childhood [17]. The presence of one autoimmune process predisposes to the development of additional autoimmune diseases. Juvenile idiopathic arthritis, though being not the most common of them, is manifested by noncharacteristic clinical features, which may delay the diagnosis [18]. This is a common reason for misdiagnosis and may confer a risk of inappropriate treatment. T1D prevalence in patients with JIA reaches up to $0.5 \%$ [16], whereas JIA-prevalence in patients with type 1 diabetes $(0.19 \%)$ is considerably greater than in the general population $(0.05 \%)$ [19]. The coexistence of a few autoimmune diseases in one patient suggests that these conditions could have a common genetic background $[5,6]$. The human leukocyte antigen (HLA), CTLA4, and PTPN22 as T-cell activation regulators are suspected of playing a key role in autoimmunity processes, i.e., the aberrant function of the molecules encoded by them may be responsible for the development of autoimmunity [20]. However, their role in JIA genetic susceptibility remains unclear [21].

Because JIA is a relatively rare disease, only a few cases of clustering JIA and type 1 diabetes were published in the last years [10-13]. The vast majority of available case reports were girls, typically for autoimmune diseases (except for T1D, where both sexes are at similar risk of the disease). In most reported patients, similarly to our cases, diabetes had been developed first, whereas juvenile rheumatoid arthritis was diagnosed afterward, sometimes followed by other autoimmune diseases [7]. Contrastingly, few studies showed that T1D developed after JIA, suggesting that T1D could have also developed because of GCS use or treatment with etanercept $[7,13,22]$. Significant problems of pediatric patients with T1D are maintaining glucose blood level stable via proper insulin dosage and managing with acute complications (hypo- and hyperglycemia). The issue is also to prevent the long-term sequelae [23-25].

Hyperglycemia and glucose variability is acknowledged as pro-inflammatory condition [26-28]. Therefore, it is crucial to avoid glycemic fluctuations in rheumatoid patients, not to stimulate and maintain inflammation, which may hinder the treatment of arthritis.

When considering the use of steroids in treating JIA in patients with concomitant T1D, one should be aware of that well-proven deleterious GCS effect on glucose metabolism $[29,30]$. It means that GCS treatment can seriously harden treatment and lead to metabolic derangement. Another critical point is an increased requirement for exogenous insulin. Insulin resistance is increased by long-term steroid use and JIA as an inflammatory process. This creates complications at the metabolic level and with the insulin dosage and administration. Chronic use of excessive insulin doses could lead to weight gain, resulting in increased insulin resistance and disturbances in triglyceride and cholesterol levels, and dyslipidemia [31-33]. As for insulin administration, high insulin doses may also implicate local side effects like increased tissue traumatization because of a bigger volume of a single injection or bolus. This can be counteracted with modern concentrated insulin types U200, U300, U500. Unfortunately, it is challenging to find an appropriate insulin pump adapted to the above-concentrated insulin formulation. The broader application of biologic drugs during the previous years has greatly reduced the need for systemic GCS in children with JIA. According to the current guidelines, the use of GCS is restricted mainly to the management of extraarticular manifestation of systemic-onset JIA or particular indications such as macrophage activation syndrome (MAS), where GCS are used intravenously in high pulse doses [34]. In subtypes other than systemic-onset JIA, GCS should be used carefully because their potential adverse effects might prevail any benefits to articular disease. A short course of low-dose prednisone (up to $0.5 \mathrm{mg} / \mathrm{kg} /$ day) may be considered in patients with severe polyarthritis refractory to other drugs or as a bridging therapy while waiting for the therapeutic effect of a recently initiated conventional or biologic DMARDs) [35]. Intra-articular glucocorticosteroid (IAC) injections remain the mainstay of therapy of oligoarticular JIA, without systemic side-effects such as the impact on glucose metabolism. Moreover, multiple IAC injections may be considered in children with polyarticular JIA to induce prompt remission of synovitis while simultaneously initiating therapy with DMARDs and biologic agents [36]. This concept seems to be especially valuable in patients with concomitant diabetes regarding better glycemic control and lower risk of metabolic derangement; however, in presented two patients, due to the need for multiple arthrocentesis and no parental consent for this approach, the IAC injections were not implemented, which resulted in a need for the use of oral GCS and subsequent poorer diabetes control.

It is worth mentioning that some patients become GCSdependent for proper JIA control; GCS have to be administrated due to recurrence of symptoms (or to be tapered slowly) [10, 13]. Notwithstanding, major therapeutic issues in JIA are not only side effects of GCS. Quality of life in JIA is deteriorated by morning stiffness, multifocal joint pain, contractures, and impaired mobility, which could result in disability in adulthood [37, 38].

Interestingly, biologic DMARDs administration in some JIA cases is associated with a significant improvement of $\mathrm{HbA} 1 \mathrm{c}$ and better long-term metabolic control of diabetes. It should probably be recommended instead of GCS when it is possible $[12,39,40]$. Also, the patients receiving biologic 
DMARDs for JIA require less insulin therapy for diabetes [18]. Moreover, DMARDs therapy is suggested to prevent T1D development in patients with rheumatoid arthritis. Therefore, it could be an argument for starting biologic DMARD therapy early in the course of JIA management to potentially ameliorate the development of other autoimmune disorders [41, 42].

Another problem worth mentioning is collagen glycation. Patients with long-lasting type 1 diabetes have a very high joint stiffness prevalence (especially hand, shoulder, and lumbar) due to the collagen glycation process and accumulation of advanced glycation end-products [43]. Although these findings were observed in adults, an association between joint stiffness and increased glycated hemoglobin might be unnoticed at a younger age. A speculative question arises whether the onset of such problems may occur already in childhood and adolescence (with a sufficiently long duration of diabetes and poor metabolic control) and become a kind of a "mask" of the morning stiffness symptom which has been often attributed to rheumatologic disease including JIA).

The psychological and psychosocial aspects of JIA and T1D as overlapping conditions and their impact on a pediatric patient are also important $[44,45]$. It is extremely difficult for a young person to cope with one chronic disease, let alone two or more. Psychological support can improve diabetes metabolic control and, consequently, affect the patient's well-being and quality of life in a long-term perspective [46]. For this reason, the clinical psychologist should be included in the interdisciplinary team taking care of such a patient. Another questionable issue remains the optimal pattern for the multidisciplinary service encompassing pediatric rheumatology, diabetes care, and transition schedule between pediatric and adult care.

It is worth mentioning that our study remains limited by a few issues. First, conclusions from our research regarding children cannot be generalized to the adult population. Second, the follow-up of our patients is considerably short (it differs in cited cases), which may bias our picture of the illness in the longer term. The following limitation is the retrospective character of the study. Because our information has been gathered from electronic medical records, our findings are limited by the possibility of gaps in the documentation that cannot be avoided despite careful data gathering.

To conclude, the treatment of a multimorbid patient requires the cooperation of several trained specialists. Diabetologists should be aware of other autoimmune diseases, including rheumatological conditions, and thus be alert to specific clinical features, e.g., joint stiffness or pain in their patients. T1D coexistence with other autoimmune conditions may deteriorate metabolic control and may considerably impede insulin treatment, especially during systemic GCS use, even despite its small dose. Hyperglycemia can worsen JIA therapy by induction and maintaining inflammation. Personal insulin pumps and CGM systems help adjust insulin dosing and maintain metabolic control during active inflammation and GCS therapy. Moreover, the broader use of IAC injections (even in polyarthritis) and earlier treatment with modern biologic drugs may reduce the need for systemic GCS. In the future, hybrid and fully closed-loop insulin pump systems may facilitate the therapy even more.

Author contributions $\mathrm{MS}, \mathrm{MO}, \mathrm{KP}$-made substantial contributions to study design and conception, acquisition, analysis, and interpretation of data and wrote the first draft of the paper. BG-O made a substantial contribution to study design and concept, acquisition, analysis, and interpretation of data and wrote and revised the paper. PA was responsible for data analysis, interpretation, and critical revision of the manuscript. JK was involved in the design, conception, analysis, and revised the paper. $\mathrm{AB}$ was involved in the design, conception, analysis, and revised the paper. All authors contributed to the discussion, read, and approved the final version of the manuscript.

Funding This research was supported by grants from the Medical University of Bialystok, Poland.

Availability of data and material The raw data supporting the conclusions of this article will be made available by the authors, without undue reservation, the medical history of the patients.

\section{Declarations}

Conflict of interest The authors declare that the research was conducted in the absence of any commercial or financial relationships that could be construed as a potential conflict of interest.

Ethics approval This work did not require the approval of the bioethics committee.

Consent to participate Patients did express due and informed consent to use their medical records for scientific purposes.

Open Access This article is licensed under a Creative Commons Attribution 4.0 International License, which permits use, sharing, adaptation, distribution and reproduction in any medium or format, as long as you give appropriate credit to the original author(s) and the source, provide a link to the Creative Commons licence, and indicate if changes were made. The images or other third party material in this article are included in the article's Creative Commons licence, unless indicated otherwise in a credit line to the material. If material is not included in the article's Creative Commons licence and your intended use is not permitted by statutory regulation or exceeds the permitted use, you will need to obtain permission directly from the copyright holder. To view a copy of this licence, visit http://creativecommons.org/licenses/by/4.0/.

\section{References}

1. Patterson CC, Harjutsalo V, Rosenbauer J et al (2018) Trends and cyclical variation in the incidence of childhood type 1 diabetes in 26 European centres in the 25 year period 1989-2013: a 
multicentre prospective registration study. Diabetol 623(62):408417. https://doi.org/10.1007/S00125-018-4763-3

2. Mobasseri M, Shirmohammadi M, Amiri T et al (2020) Prevalence and incidence of type 1 diabetes in the world: a systematic review and meta-analysis. Heal Promot Perspect 10:98-115. https://doi.org/10.34172/hpp.2020.18

3. Lin X, Xu Y, Pan X et al (2020) Global, regional, and national burden and trend of diabetes in 195 countries and territories: an analysis from 1990 to 2025. Sci Rep 10:14790. https://doi.org/10. 1038/s41598-020-71908-9

4. Williams R, Karuranga S, Malanda B et al (2020) Global and regional estimates and projections of diabetes-related health expenditure: results from the International Diabetes Federation Diabetes Atlas, 9th edition. Diabetes Res Clin Pract 162:108072. https://doi.org/10.1016/j.diabres.2020.108072

5. Hinks A, Barton A, John S et al (2005) Association between the PTPN22 gene and rheumatoid arthritis and juvenile idiopathic arthritis in a UK population: further support that PTPN22 is an autoimmunity gene. Arthritis Rheum 52:1694-1699. https://doi. org/10.1002/art.21049

6. Burn GL, Svensson L, Sanchez-Blanco C et al (2011) Why is PTPN22 a good candidate susceptibility gene for autoimmune disease? FEBS Lett 585:3689-3698. https://doi.org/10.1016/j. febslet.2011.04.032

7. Ben-Skowronek I, Michalczyk A, Piekarski R et al (2013) Type III polyglandular autoimmune syndromes in children with type 1 diabetes mellitus. Ann Agric Environ Med 20:140-146

8. Mayer-Davis EJ, Kahkoska AR, Jefferies C et al (2018) ISPAD clinical practice consensus guidelines 2018: definition, epidemiology, and classification of diabetes in children and adolescents. Pediatr Diabetes. https://doi.org/10.1111/pedi.12773

9. Petty RE, Southwood TR, Manners P et al (2004) International League of Associations for Rheumatology Classification of juvenile idiopathic arthritis: second revision, Edmonton, 2001. J Rheumatol 31:390-392

10. Mroczkowska-Juchkiewicz A, Postẹpski J, Olesińska E et al (2017) Exceptional manifestation of polyautoimmunity in a very young girl-a case report. Cent Eur J Immunol 42:107-110. https://doi.org/10.5114/ceji.2017.67324

11. Nagy KH, Lukacs K, Sipos P et al (2010) Type 1 diabetes associated with Hashimoto's thyroiditis and juvenile rheumatoid arthritis: a case report with clinical and genetic investigations. Pediatr Diabetes 11:579-582. https://doi.org/10.1111/j.1399-5448.2010. 00676.x

12. Olivieri AN, Iafusco D, Mellos A et al (2013) Refractory rheumatoid factor positive polyarthritis in a female adolescent already suffering from type 1 diabetes mellitus and Hashimoto's thyroiditis successfully treated with etanercept. Ital J Pediatr. https://doi. org/10.1186/1824-7288-39-64

13. Nolfi-Donegan D, Viswanathan A, Chefitz D, Moorthy L (2012) Case report of a child with recently diagnosed diabetes mellitus type 1 and subsequent systemic arthritis. Internet J Pediatr Neonatol 14(1). ISSN: 1528-8374.9

14. Araszkiewicz A, Bandurska-Stankiewicz E, Borys S et al (2021) 2021 Guidelines on the management of patients with diabetes. A position of Diabetes Poland. Clin Diabetol 10:1-113. https://doi. org/10.5603/dk.2021.0001

15. Wallace CA, Giannini EH, Huang B, Childhood Arthritis Rheumatology Research Alliance; Pediatric Rheumatology Collaborative Study Group; Paediatric Rheumatology International Trials Organisation et al (2011) American College of Rheumatology provisional criteria for defining clinical inactive disease in select categories of juvenile idiopathic arthritis. Arthritis Care Res (Hoboken) 63:929-936. https://doi.org/10.1002/acr.20497

16. Consolaro A, Ruperto N, Bracciolini G, Paediatric Rheumatology International Trials Organization (PRINTO) et al (2014)
Defining criteria for high disease activity in juvenile idiopathic arthritis based on the juvenile arthritis disease activity score. Ann Rheum Dis 73:1380-1383. https://doi.org/10.1136/annrh eumdis-2013-204186

17. Ziegler R, Neu A (2018) Diabetes in childhood and adolescencea guideline-based approach to diagnosis, treatment, and follow-up. Dtsch Arztebl Int 115:146-156. https://doi.org/10.3238/arztebl. 2018.0146

18. Schenck S, Rosenbauer J, Niewerth M et al (2018) Comorbidity of type 1 diabetes mellitus in patients with juvenile idiopathic arthritis. J Pediatr 192:196-203. https://doi.org/10.1016/j.jpeds. 2017.07.050

19. Hermann G, Thon A, Mönkemöller K et al (2015) Comorbidity of type 1 diabetes and juvenile idiopathic arthritis. J Pediatr 166:930935.e3. https://doi.org/10.1016/j.jpeds.2014.12.026

20. Brand O, Gough S, Heward J (2005) HLA, CTLA-4 and PTPN22: the shared genetic master-key to autoimmunity? Expert Rev Mol Med 7:1-15. https://doi.org/10.1017/S1462 399405009981

21. Seldin MF, Shigeta R, Laiho K et al (2005) Finnish case-control and family studies support PTPN22 R620W polymorphism as a risk factor in rheumatoid arthritis, but suggest only minimal or no effect in juvenile idiopathic arthritis. Genes Immun 68(6):720 722. https://doi.org/10.1038/sj.gene.6364255

22. Tack CJ, Kleijwegt FS, Van Riel PLCM, Roep BO (2009) Development of type 1 diabetes in a patient treated with anti-TNF- $\alpha$ therapy for active rheumatoid arthritis. Diabetologia 52:14421444. https://doi.org/10.1007/s00125-009-1381-0

23. White NH (2015) Long-term outcomes in youths with diabetes mellitus. Pediatr Clin N Am 62:889-909. https://doi.org/10. 1016/j.pcl.2015.04.004

24. Pastore I, Bolla AM, Montefusco L et al (2020) The impact of diabetes mellitus on cardiovascular risk onset in children and adolescents. Int J Mol Sci 21:1-17. https://doi.org/10.3390/IJMS2 1144928

25. Jones S, Khanolkar AR, Gevers E et al (2019) Cardiovascular risk factors from diagnosis in children with type 1 diabetes mellitus: a longitudinal cohort study. BMJ Open Diabetes Res Care 7:e000625. https://doi.org/10.1136/BMJDRC-2018-000625

26. Gyurko R, Siqueira CC, Caldon N et al (2006) Chronic hyperglycemia predisposes to exaggerated inflammatory response and leukocyte dysfunction in Akita mice. J Immunol 177:7250-7256. https://doi.org/10.4049/jimmunol.177.10.7250

27. Kwan CK, Fu SC, Yung PS (2020) A high glucose level stimulate inflammation and weaken pro-resolving response in tendon cells-a possible factor contributing to tendinopathy in diabetic patients. Asia Pac J Sports Med Arthrosc 19:1-6. https://doi.org/ 10.1016/j.asmart.2019.10.002

28. Wellen KE, Hotamisligil GS (2005) Inflammation, stress, and diabetes. J Clin Invest 115:1111-1119. https://doi.org/10.1172/ JCI25102

29. Patel R, Williams-Dautovich J, Cummins CL (2014) Minireview: new molecular mediators of glucocorticoid receptor activity in metabolic tissues. Mol Endocrinol 28:999. https://doi.org/10. 1210/ME.2014-1062

30. Pasieka AM, Rafacho A (2016) Impact of glucocorticoid excess on glucose tolerance: clinical and preclinical evidence. Metabolites. https://doi.org/10.3390/METABO6030024

31. Szadkowska A, Pietrzak I, Mianowska B et al (2008) Insulin sensitivity in type 1 diabetic children and adolescents. Diabet Med 25:282-288. https://doi.org/10.1111/j.1464-5491.2007.02357.x

32. Sinaiko AR, Jacobs DR, Steinberger J et al (2001) Insulin resistance syndrome in childhood: associations of the euglycemic insulin clamp and fasting insulin with fatness and other risk factors. $\mathbf{J}$ Pediatr 139:700-707. https://doi.org/10.1067/mpd.2001.118535 
33. Heliövaara MK, Teppo AM, Karonen SL et al (2005) Plasma IL-6 concentration is inversely related to insulin sensitivity, and acutephase proteins associate with glucose and lipid metabolism in healthy subjects. Diabetes Obes Metab 7:729-736. https://doi.org/ 10.1111/j.1463-1326.2004.00463.x

34. Ringold S, Weiss PF, Beukelman T et al (2013) American Collge of Rheumatology. 2013 update of the 2011 American College of Rheumatology recommendations for the treatment of juvenile idiopathic arthritis: recommendations for the medical therapy of children with systemic juvenile idiopathic arthritis and tuberculosis screening among children receiving biologic medications. Arthritis Rheum 65:2499-2512. https://doi.org/10.1002/art.38092

35. Ringold S, Angeles-Han ST, Beukelman T et al (2019) 2019 American College of Rheumatology/Arthritis Foundation Guideline for the Treatment of Juvenile idiopathic arthritis: therapeutic approaches for non-systemic polyarthritis, sacroiliitis, and enthesitis. Arthritis Care Res (Hoboken) 71:717-734. https://doi.org/10. 1002/acr.23870

36. Scott C, Meiorin S, Filocamo G et al (2010) A reappraisal of intra-articular corticosteroid therapy in juvenile idiopathic arthritis. Clin Exp Rheumatol 28:774-781

37. Hyrich KL, Lal SD, Foster HE et al (2010) Disease activity and disability in children with juvenile idiopathic arthritis one year following presentation to paediatric rheumatology. Results from the Childhood Arthritis Prospective Study. Rheumatology 49:116-122. https://doi.org/10.1093/RHEUMATOLOGY/ KEP352

38. Moorthy LN, Peterson MG, Hassett AL, Lehman TJ (2010) Burden of childhood-onset arthritis. Pediatr Rheumatol 81(8):1-9. https://doi.org/10.1186/1546-0096-8-20

39. Mantravadi S, George M, Brensinger C et al (2020) Impact of tumor necrosis factor inhibitors and methotrexate on diabetes mellitus among patients with inflammatory arthritis. BMC Rheumatol. https://doi.org/10.1186/S41927-020-00138-3
40. Conigliaro P, Triggianese P, De Martino E et al (2019) Challenges in the treatment of rheumatoid arthritis. Autoimmun Rev. https:// doi.org/10.1016/j.autrev.2019.05.007

41. Antohe J, Bili A, Sartorius JA et al (2012) Diabetes mellitus risk in rheumatoid arthritis: reduced incidence with anti-tumor necrosis factor $\alpha$ therapy. Arthritis Care Res (Hoboken) 64:215-221. https://doi.org/10.1002/acr.20657

42. Chandrakasan S, Prahalad S (2018) Revisiting Type 1 diabetes as a comorbidity in patients with juvenile idiopathic arthritis. J Pediatr 192:6-7. https://doi.org/10.1016/j.jpeds.2017.09.067

43. Holte KB, Gunnar Juel N, Brox JI et al (2017) Hand, shoulder and back stiffness in long-term type 1 diabetes; cross-sectional association with skin collagen advanced glycation end-products. The Dialong study. J Diabetes Complicat 31:1408-1414. https:// doi.org/10.1016/j.jdiacomp.2017.06.007

44. Frank MR (2005) Psychological issues in the care of children and adolescents with type 1 diabetes. Paediatr Child Health 10:18-20

45. Delamater AM, de Wit M, McDarby V et al (2018) ISPAD Clinical Practice Consensus Guidelines 2018: psychological care of children and adolescents with type 1 diabetes. Pediatr Diabetes 19:237-249. https://doi.org/10.1111/pedi.12736

46. Galler A, Hilgard D, Bollow E et al (2020) Psychological care in children and adolescents with type 1 diabetes in a real-world setting and associations with metabolic control. Pediatr Diabetes 21:1050-1058. https://doi.org/10.1111/pedi.13065

Publisher's Note Springer Nature remains neutral with regard to jurisdictional claims in published maps and institutional affiliations. 